\title{
Is twice the duration of washout sufficient time between multiple breath nitrogen washout tests?
}

\author{
To the Editor:
}

Multiple breath washout (MBW) assesses both lung volume (functional residual capacity (FRC)) and ventilation inhomogeneity (lung clearance index (LCI)). The latter's insight into underlying lung pathology and response to intervention has triggered a recent MBW resurgence [1-4]. Validated commercial equipment has become available [5], increasing suitability for widespread use, and a European Respiratory Society/American Thoracic Society endorsed MBW consensus statement has been published to guide manufacturers and operators [6]. This document highlights the need for further evidence across several recommendation areas. In the May 2015 issue of this journal SALAMON et al. [7] tackled one such important issue: what duration should be employed between two MBW tests within a testing session? Previous recommendations of gaps of $\geqslant 15 \mathrm{~min}$ in healthy and $\leqslant 60 \mathrm{~min}$ in obstructed patients [8] were shown to be too conservative. Using a gap of the previous washout duration led to $10 \%$ lower FRC values $(\mathrm{p}<0.001)$ but no effect was observed if this was doubled, in line with the recent consensus-based recommendation. [9]. The results presented here address two important limitations to the data presented by SALAmon et al. [7], as acknowledged by the authors themselves, to further strengthen the recommendation's evidence base. First, in their paediatric cohort, an alternate, fixed-gap duration protocol was used (5 and $15 \mathrm{~min}$ ), which translated to an excessive duration between tests (mean 2.6x). Secondly, LCI data was not reported, which may have compromised the ability to detect any detrimental effects.

In the setting of ventilation inhomogeneity, insufficient wash-in of nitrogen between nitrogen-based MBW tests affects poorly ventilated lung units to a greater degree and may lead to an incomplete contribution by these units during subsequent tests. Contributions by these poorly ventilated units are weighted towards the latter portion of the washout curve, which contains the end threshold for LCI determination. By contrast, FRC calculation, based on cumulative expired nitrogen volume, has larger relative contributions during initial portions of the washout with diminishing contributions as washout progresses and end-tidal nitrogen concentration falls.

We performed a retrospective analysis of paediatric nitrogen MBW tests at our tertiary paediatric institution. Data were collated from 90 children (37 with cystic fibrosis, 41\%) equally split across three paediatric age ranges (preschool, early school age and adolescence). Consecutive technically acceptable tests were included, where possible in triplicate to examine for cumulative effects over the three tests. No medication was given prior to testing and testing occurred during clinical stability. No specific breathing manoeuvres were used between tests to facilitate nitrogen wash-in. The commercial equipment used (Ecomedics Exhalyzer D; Eco Medics AG, Dürnten, Switzerland) employed a lock-out time of $1.5 \times$ the preceding washout duration before the software could be advanced towards the next test. Actual duration between tests was calculated to capture additional time to advance the software and settle the child back on MBW equipment such that data collection could recommence. A generalised estimating equation was used to investigate FRC and LCI change over time. Ethical approval was granted as a quality control project.

The cohort represented both a wide range of FRC $(0.31-4.31 \mathrm{~L})$ and ventilation inhomogeneity (LCI $5.90-17.05$ ) (table 1). Overall actual mean \pm SD time between washouts was $1.91 \pm 0.24 \times$ the previous washout time and did not differ between age groups. In all cases, baseline end-tidal nitrogen values had returned to within $1.5 \%$ (relative) of the first test's starting end-tidal nitrogen concentration (acceptability

@ERSpublications

A wait time between MBNW tests of twice the washout duration has no detrimental effect on LCI and FRC outcomes http://ow.ly/RF8y304V3PB

Cite this article as: Hardaker KM, Gustafsson P, Cooper $\mathrm{P}$, et al. Is twice the duration of washout sufficient time between multiple breath nitrogen washout tests?. Eur Respir J 2017; 49: 1501832 [https:// doi.org/10.1183/13993003.01832-2015]. 
TABLE 1 Baseline data of the cohort and summary of differences in multiple breath washout parameters classified according to age

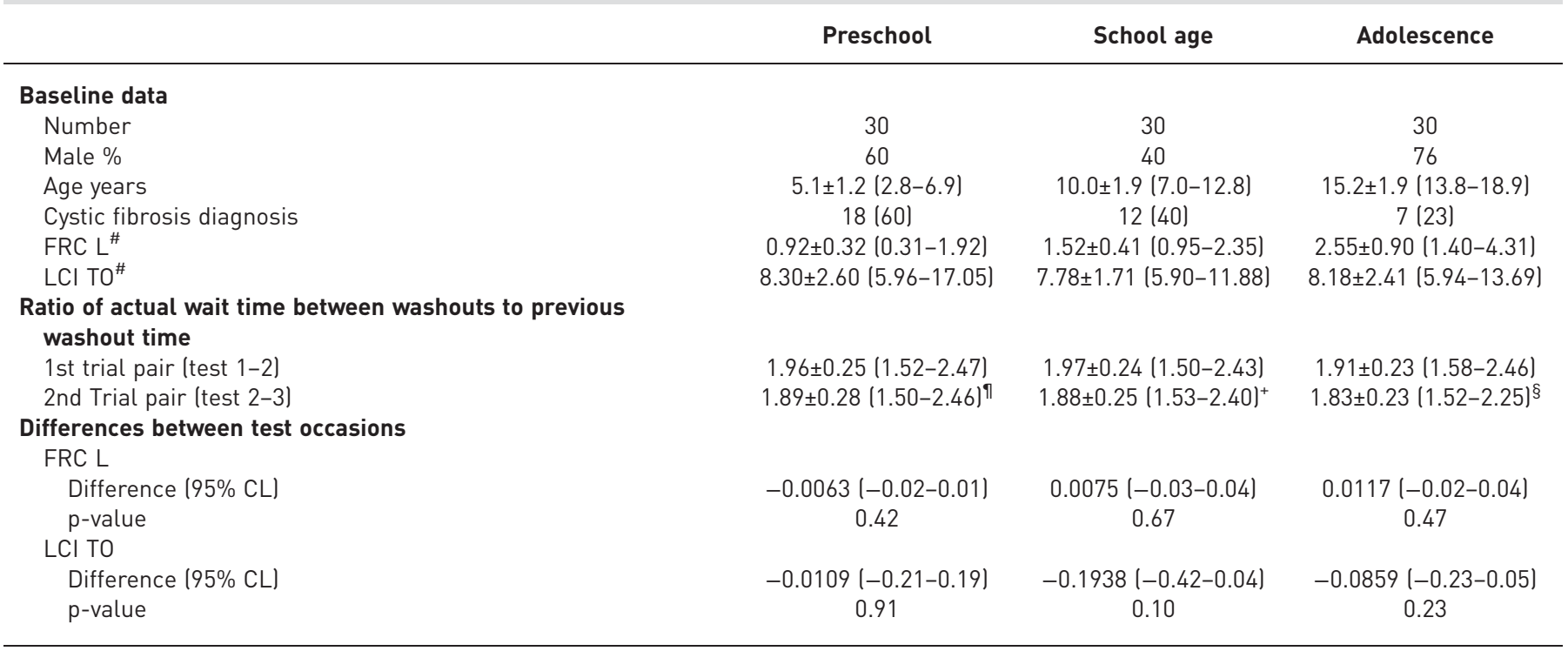

Data are presented as $\mathrm{n}, \mathrm{n}(\%)$ and mean \pm SD (range), unless otherwise stated. \#: based on the mean result from three technically acceptable tests during that testing session; ${ }^{\uparrow}: n=17 ;{ }^{+}: n=15 ;{ }^{\S}: n=23$. CL: confidence limits; FRC: functional residual capacity; LCl: lung clearance index; TO: lung turnovers.

criteria determined by software). No statistically significant difference in LCI and FRC across test pairs or triplicates was found. The magnitude of difference (as a percentage of baseline value) did not increase as disease severity (or LCI) increased (data not shown).

The data presented here build on the important work of SALAMON et al. [7] to further support the consensus recommendation of twice the previous washout time to ensure adequate wash-in of nitrogen during room air breathing with nitrogen MBW [9]. We used an approach based solely on previous washout duration, in larger subject numbers across the entire paediatric age range and reported both FRC and LCI values. Applicability across both a wide range of underlying lung volumes and severity of ventilation inhomogeneity (LCI $\leqslant 17$ ) was examined. No detrimental effect on either outcome was observed, either between tests or across triplicate test sessions. Applicability to MBW use in very severe ventilation inhomogeneity (LCI >17) is unclear, but in these subjects the long testing time required remains a significant barrier to routine clinical use. The lack of effect seen at the lower end of the washout durations (towards $1.5 \times$ washout duration) suggests that future work should focus on this lower threshold to reduce total test session duration further. Future work to reduce the gap between tests should also explore the utility of defining a minimum threshold for starting end-tidal nitrogen prior to commencing the next washout test. Such an approach was not examined as part of this analysis.

Kate M. Hardaker ${ }^{1}$, Per Gustafsson ${ }^{2}$, Peter Cooper $^{1}$, Dominic Fitzgerald ${ }^{1,3}$, Hiran Selvadurai ${ }^{1,3}$ and Paul D. Robinson ${ }^{1,3}$ ${ }^{1}$ Dept of Respiratory Medicine, The Children's Hospital at Westmead, Westmead, Australia. ${ }^{2}$ Central Hospital, Skövde, Sweden. ${ }^{3}$ The Children's Hospital at Westmead Clinical School, Discipline of Paediatrics and Child Health, Faculty of Medicine, University of Sydney, Sydney, Australia.

Correspondence: Paul D. Robinson, Dept of Respiratory Medicine, The Children's Hospital at Westmead, Locked Bag 4001, Westmead, NSW, 2145 Australia. E-mail: paul.robinson1@health.nsw.gov.au

Received: Nov 042015 | Accepted after revision: Sept 232016

Support statement: This study was supported by Cystic Fibrosis Fundraising. Funding information for this article has been deposited with the Open Funder Registry.

Conflict of interest: None declared.

\section{References}

1 Amin R, Subbarao P, Jabar A, et al. Hypertonic saline improves the LCI in paediatric patients with CF with normal lung function. Thorax 2010; 65: 379-383.

2 Amin R, Subbarao P, Lou W, et al. The effect of dornase alfa on ventilation inhomogeneity in patients with cystic fibrosis. Eur Respir J 2011; 37: 806-812. 
3 Aurora P, Bush A, Gustafsson P, et al. Multiple-breath washout as a marker of lung disease in preschool children with cystic fibrosis. Am J Respir Crit Care Med 2005; 171: 249-256.

4 Aurora P, Gustafsson P, Bush A, et al. Multiple breath inert gas washout as a measure of ventilation distribution in children with cystic fibrosis. Thorax 2004; 59: 1068-1073.

5 Singer F, Houltz B, Latzin P, et al. A realistic validation study of a new nitrogen multiple-breath washout system. PLoS One 2012; 7: e36083.

6 Robinson PD, Latzin P, Verbanck S, et al. Consensus statement for inert gas washout measurement using multipleand single-breath tests. Eur Respir J 2013; 41: 507-522.

7 Salamon ER, Gain KR, Hall GL. Defining the appropriate waiting time between multiple-breath nitrogen washout measurements. Eur Respir J 2015; 45: 1489-1491.

8 Wanger J, Clausen JL, Coates A, et al. Standardisation of the measurement of lung volumes. Eur Respir J 2005; 26: 511-522.

9 Robinson PD, Latzin P, Verbanck S, et al. ERS/ATS consensus statement for inert gas washout measurement using multiple and single breath tests. Eur Respir J 2013; 41: 507-522.

Copyright @ERS 2017

From the authors:

We read the paper by K.M. Hardaker and co-workers with great interest. Their study confirmed our earlier data: that a washout-derived wait time between measurements of nitrogen-based multiple breath washout (MBW) was not influenced by disease severity, and that on average a wait time of twice the duration of the washout between nitrogen MBW tests eliminated potential measurement artefacts [1].

K.M. Hardaker and co-workers investigated whether our recommendation to wait at least twice the initial washout time between nitrogen MBW measurements was applicable in a larger cohort of children, with a retrospective analysis of clinically obtained data. In our study, we initially used a combination of wait times ( 5 and $15 \mathrm{~min}$ ), which were randomised, in children aged 7 years and older. Our protocol was subsequently reviewed for an adult dataset and a wait time based on a function of initial washout time was used. The clinical data set used by Hardaker and co-workers allowed the expression of wait time in relation to washout time, rather than a fixed time, and this was, on average, 1.9 times the previous washout time. We commend their inclusion of preschool-aged children and are pleased that our recommendation is supported in this younger group by their evidence, which showed that a wait time of 1.50-2.27 times the previous washout time was sufficient for repeat measurements of functional residual capacity (FRC) to return reproducible values. A limitation of this retrospective analysis was that, unlike in our study, the time between repeat trials was not randomised and therefore an order effect could not be excluded. Similarly, while their data confirms that a wait time of twice the washout duration was sufficient to avoid artefacts in young children, their study was not designed to specifically test if shorter wait times were appropriate.

Hardaker and co-workers added value to this work by including the lung clearance index (LCI) and found that waiting $\sim 2$ times the previous washout time ensured repeat measurements were reproducible. While our study did not report LCI, we are confident that any potentially detrimental effects of ventilation inhomogeneity on the measured FRC were accounted for by a wait time that was equal to, or exceeded, twice that of the initial washout time. This is supported by our observation in an adult cohort that a wait time based on the patient's own washout time was appropriate in obstructive lung disease. Random-effects regression analysis demonstrated no independent effect of obstructive disease severity on the washout time ( $p=0.98)$.

Hardaker and co-workers commented that future work could be done towards pursuing a reduced washout time; however, investigations should proceed with caution as the shorter the time between measurements the greater the risk of effects due to ventilation inhomogeneity. Although no recorded effects were seen on repeat FRC measurement after waiting only once the initial washout time in both our healthy and restricted groups, our obstructive lung disease group exhibited a significant difference in FRC. This suggests that reduced wait times may lead to measurement inaccuracies, although we do advocate further optimisation of lung-function testing protocols to improve the clinical reliability of the test.

@ERSpublications

Waiting for multiple breath washout: when is long enough? http://ow.ly/5b5b306mYYh

Cite this article as: Salamon ER, Gain KR, Hall GL. Is twice the duration of washout sufficient time between multiple breath nitrogen washout tests?. Eur Respir J 2017; 49: 1602064 [https://doi.org/10.1183/ 13993003.02064-2016]. 
The most recent consensus statement for inert gas washout measurements recommends that three technically acceptable FRC measurements are obtained with $<10 \%$ variation from the minimum to maximum values [2]. The work of Hardaker and co-workers, in addition to our own work, means that this recommendation is attainable within a reasonable time period in a clinical laboratory. We welcome this contribution that has added to the body of work on nitrogen MBW testing procedures by including a younger cohort as well as LCI in the analysis of wait time effect on repeated nitrogen MBW measurements. This work supports our recommendation to wait twice the initial washout time between repeat measurements of FRC and we continue to strongly encourage the development of evidence-based guidelines to inform best practice in the clinical lung-function laboratory.

Elizabeth R. Salamon ${ }^{1}$, Kevin R. Gain ${ }^{1}$ and Graham L. Hall ${ }^{2,3,4}$

${ }^{1}$ Respiratory Medicine, Royal Perth Hospital, Perth, Australia. ${ }^{2}$ Children's Lung Health, Telethon Kids Institute, Perth, Australia. ${ }^{3}$ School of Physiotherapy and Exercise Science, Curtin University, Perth, Australia. ${ }^{4}$ Centre for Child Health Research, University of Western Australia, Perth, Australia.

Correspondence: Graham L. Hall, Telethon Kids Institute, Children's Lung Health, PO Box 855, West Perth, Western Australia 6872, Australia. E-mail: graham.hall@telethonkids.org.au

Received: Oct 212016 | Accepted after revision: Oct 212016

Conflict of interest: None declared.

\section{References}

1 Salamon ER, Gain KR, Hall GL. Defining the appropriate waiting time between multiple-breath nitrogen washout measurements. Eur Respir J 2015; 45: 1489-1491.

2 Robinson PD, Latzin P, Verbanck S, et al. Consensus statement for inert gas washout measurement using multipleand single-breath tests. Eur Respir J 2013; 41: 507-522. 